\title{
Discrimination of signal oscillations in the in vitro olfactory bulb with microelectrode array
}

\author{
CHEN QingMei ${ }^{1,2}$, DONG Qi $^{1}$, HU Liang $^{1}$, LING ShuCai $^{3}$, YIN YiFei $^{3}$, LIU QingJun $^{1} \&$ \\ WANG Ping $^{1 *}$ \\ ${ }^{1}$ Biosensor National Special Laboratory, Key Laboratory of Biomedical Engineering of Ministry of Education, Department of Biomedical \\ Engineering, Zhejiang University, Hangzhou 310027, China; \\ ${ }^{2}$ Department of Biomedical Engineering, Jiangxi University of Traditional Chinese Medicine, Nanchang 330006, China; \\ ${ }^{3}$ Institute of Anatomy and Cell Biology, School of Medicine, Zhejiang University, Hangzhou 310058, China
}

Received July 1, 2012; accepted October 7, 2012; published online July 2, 2013

\begin{abstract}
The aim of the present study was to investigate the electrophysiological characteristics of the different layers of the olfactory bulb $(\mathrm{OB})$. We used an in vitro $\mathrm{OB}$ slice coupled onto a microelectrode array (MEA) for simultaneous detection of spontaneous activities of OB neurons at different sites. Different frequency oscillations dominated the different layers of the OB slice, and the gamma frequency oscillations mainly appeared in the glomerular layer. The waves consisted of negative, positive, and bidirectional spikes, and were distributed at the different layers of the OB slice. Thus, combination of the OB slice with MEA is a useful technique for identifying signal oscillations by multi-site synchronous measurement, and will allow further studies on olfactory information coding and processing function.
\end{abstract}

olfactory bulb slice, signal oscillation discrimination in vitro, microelectrode array

Citation: $\quad$ Chen Q M, Dong Q, Hu L, et al. Discrimination of signal oscillations in the in vitro olfactory bulb with microelectrode array. Chin Sci Bull, 2013, 58: 3015-3018, doi: 10.1007/s11434-013-5934-8

Fast oscillatory neuronal network activity is an important feature of information processing in multiple brain regions including sensory [1], motor [2], and perceptive [3] circuits. The olfactory bulb (OB) has also been reported to exhibit electrophysiological activity, including signal oscillations such as theta [4,5], alpha [6], beta [6], and gamma [7-10] band waves. Previous studies calculated power spectral densities to examine the frequency distribution of $\mathrm{OB}$ neuron responses, as well as their role in the olfactory information processing $[7,9,10]$. Fast gamma frequency oscillations are widely considered to be crucial for odor discrimination [9], while the theta rhythm is considered important for temporally reformatting the strengths and patterns of synaptic input in the olfactory system [4], and also indicates the rhythm of inhalation [5]. However, the different types of oscillations that exist in the $\mathrm{OB}$ and their localization to

*Corresponding author (email: cnpwang @ zju.edu.cn) different OB layers are unknown.

In the present study, we recorded spontaneous signals of the OB slice using a microelectrode array (MEA) for synchronous multi-site measurement, and examined layer specific oscillation characteristics.

\section{Materials and methods}

\subsection{Slice preparation}

Animal experiments were performed according to the guidelines of Zhejiang provincial management committee of medical experiment animals. Wistar rats of either sex (approximately 3 weeks old) were anesthetized using 20\% urethane $(7 \mathrm{~mL} / \mathrm{kg})$ and then decapitated. Preparation of artificial cerebrospinal fluid (ACSF) and tissue slices were performed as previously reported [11]. In brief, horizontal slices $(300 \mu \mathrm{m}$ thick) were cut with a vibratome (Vibratome 1000 Plus; 
(a)

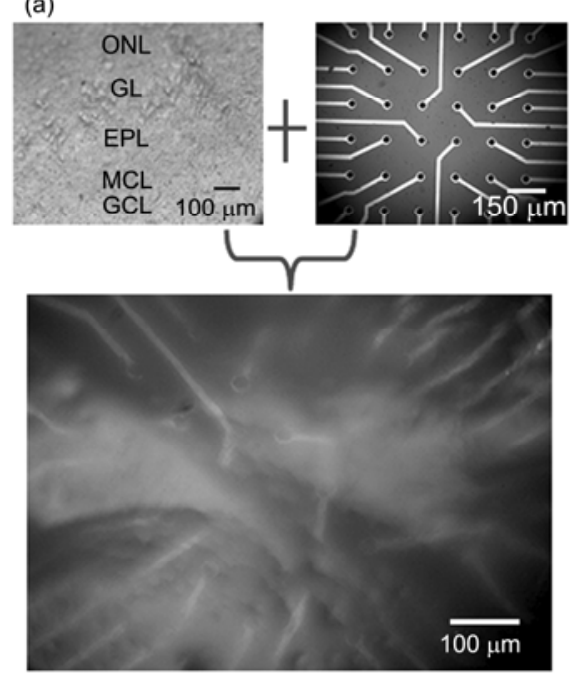

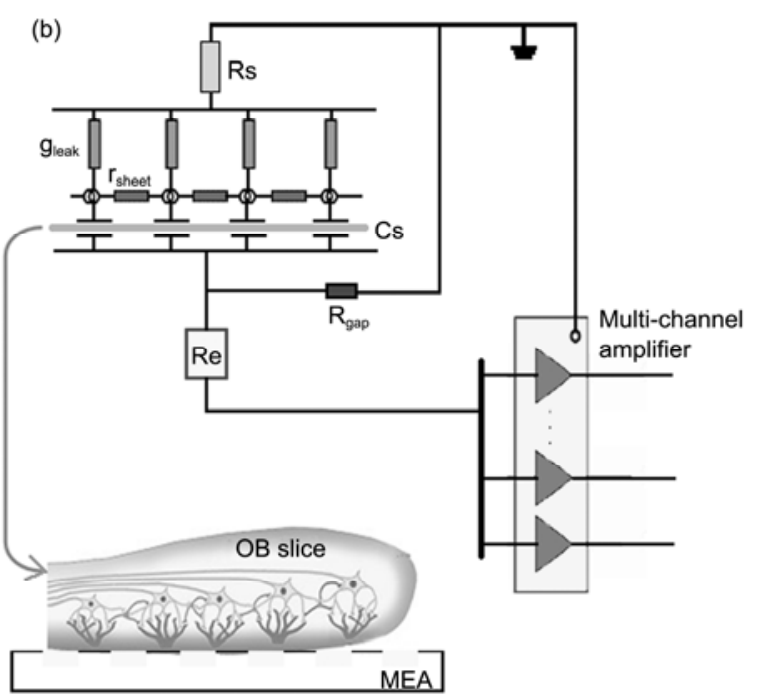

Figure 1 (a) Fabrication of the OB slice-based biosensor. (b) Sketch map of testing system. EPL: external plexiform layer, GCL: granule cell layer, GL: glomerular layer, MCL: mitral cell layer, ONL: olfactory nerve layer.

Vibratome Company, USA). For electrophysiological measurements, slices were incubated in ACSF continuously bubbled with a mixture of $95 \% \mathrm{O}_{2}$ and $5 \% \mathrm{CO}_{2}$ at room temperature for about $1 \mathrm{~h}$. To accurately define the layers of the OB slice, Nissl staining was used to dye the slice after measurement.

\subsection{Signal sampling and processing}

To couple the OB slice onto the MEA, we created a biological-electrical sensing system that allowed bidirectional communication between the controlling circuit and the biological system (Figure 1(a)). Using this OB slice-based biosensor, the electrophysiological activities of the $\mathrm{OB}$ can be synchronously detected at multiple sites. A schematic of the signal measuring system is shown in Figure 1(b), which was arranged in a shielding box to reduce power-line interference. Data acquisition was performed via a multi-channel system (MED16; Multi Channel Systems MCS GmbH, Germany), which allowed 16-channel synchronous real-time detection. During signal measurement, the sampling frequency was set at $10 \mathrm{kHz}$. To investigate the oscillation characteristics of the recorded signals at different layers of the OB slice, signals were processed with band-pass filtering in five bands: delta, 1-3 Hz; theta, 4-7 Hz; alpha, 8-13 $\mathrm{Hz}$; beta, 14-29 Hz; and gamma, 30-115 Hz.

\section{Results}

\subsection{Olfactory bulb slice modality}

Neurons in the mammalian OB are arranged regularly, and form a layered-structure comprising the olfactory nerve layer (ONL), glomerular layer (GL), external plexiform layer
(EPL), mitral cell layer (MCL), and granule cell layer (GCL). An example of an OB slice stained with Nissl showing the various layers is shown in Figure 2. The presence of these layers indicated that the OB slice is viable and can be used for electrophysiological recordings.

\subsection{Spontaneous signals}

Using our testing system (Figure 1(b)), multi-point data can be acquired simultaneously with the multi-channel amplifier. Neuronal spontaneous activities recorded from the ONL, GL, EPL, MCL, and GCL of the OB slice are shown in Figure 3(a). The spikes in the ONL and the GL were mainly negative. However, positive waves predominated in the MCL and GCL, while both the negative and positive spikes appeared in the EPL. The amplitude and frequency of the signals differed between the different layers of the OB. The maximal amplitude of the spontaneous responses was approximately $200 \mu \mathrm{V}$. The spike polarity distribution recorded using this system was similar to that previously reported

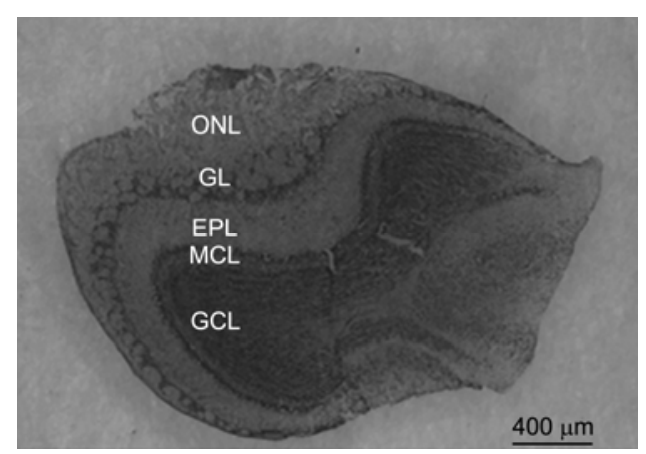

Figure 2 Photomicrograph of the OB slice stained with Nissl showing presence of multiple layers. 


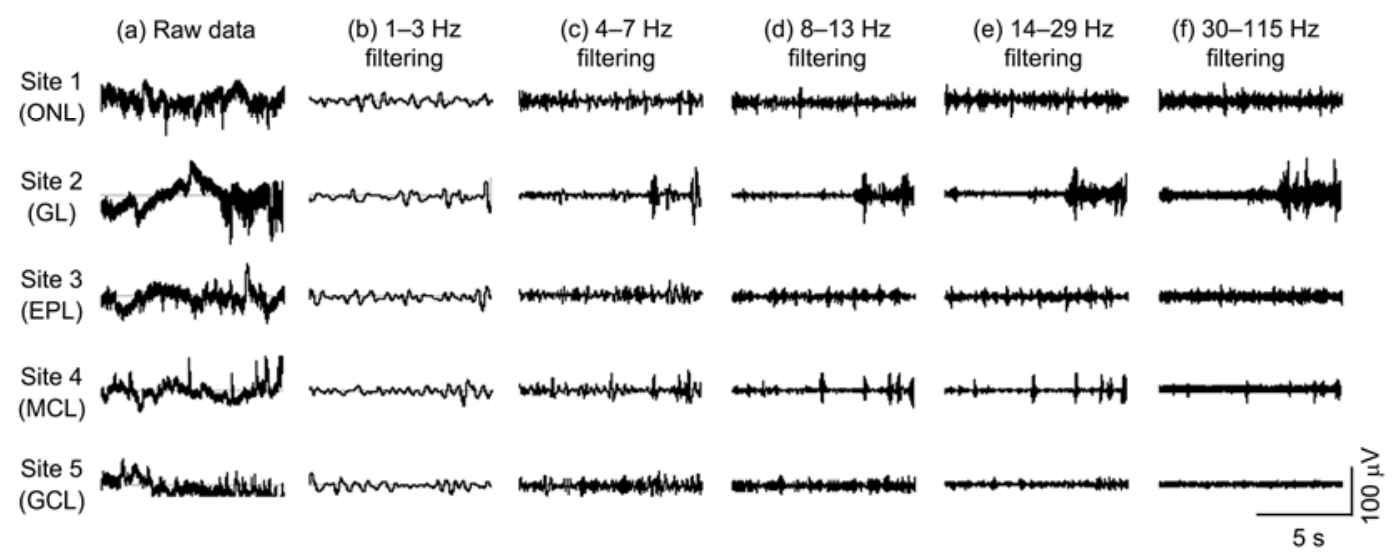

Figure 3 Raw data (a) and filtered signals (b)-(f) in bands of (b) $1-3 \mathrm{~Hz}$, (c) $4-7 \mathrm{~Hz}$, (d) $8-13 \mathrm{~Hz}$, (e) 14-29 Hz, and (f) $30-115 \mathrm{~Hz}$ recorded from the ONL, GL, EPL, MCL and GCL, respectively, in the OB slice.

using the patch clamp technique [12].

\subsection{Filtered signals in different bands}

Raw data were processed by band-pass filtering to examine the differences in oscillating characteristics of the signals at the different layers of the OB slice. Each layer consistently produced a specific pattern of signals. The band-pass filtering results from eight experiments ( $n=5$ rats per group) in the five different frequency bands are shown in Figure 3(b)-(f). Delta $(1-3 \mathrm{~Hz})$, theta $(4-7 \mathrm{~Hz})$, and alpha (8-13 $\mathrm{Hz}$ ) frequency waves appeared in nearly all layers of the OB slice, while gamma oscillation (30-115 Hz) mainly existed in the GL. In the ONL, the signal comprised nearly all frequency waves, while in the EPL and MCL the waves included the theta, alpha, and beta (14-29 Hz) frequency oscillations. In the GCL, the lower frequency $(4-13 \mathrm{~Hz})$ dominated the oscillations.

\section{Discussion}

In the present study, we found that the five different layers of in vitro $\mathrm{OB}$ slices exhibited spontaneous neuronal activity with different characteristics, including the amplitude, frequency, and the spike polarity. These data suggest that use of the MEA allows synchronous measurement of neuronal electrophysiology properties at different sites of the OB slice. A negative current is typically caused by cellular depolarization with ionic current influx [13]. Both negative and positive spikes are present in layered structures, which represent the active zone (sink) and the passive zone (source), respectively, of the current [13-15]. Thus, according to spike polarity (Figure 3), the ONL and GL are the current receivers, while the MCL and GCL are the current emitters; i.e., signals within the layered OB slice may propagate from the ONL and GL to the MCL and GCL through the EPL.

Delta, theta, and alpha oscillation were present in all lay- ers of the in vitro $\mathrm{OB}$ slice, suggesting that these waves are unlikely related to the specific physiological function of each layer. By contrast, the GL exhibited signals with high frequency oscillations, particularly the gamma oscillation. Thus, signals in the GL may be highly processed, which is considered important for odor discrimination [9].

Overall, our data suggest that the combination of the OB slice with MEA is an effective bio-sensing system for detection of neuronal extracellular potentials and identification of the signal oscillation characteristics of the in vitro $\mathrm{OB}$ slice. However, this system requires further characterization, as the extracellular conditions in vitro may not accurately represent that in vivo. Nevertheless, this model may be useful for further elucidation of odor discrimination and olfactory information encoding. Future studies are required to compare the responses observed in this system to those in vivo.

This work was supported by the National Natural Science Foundation of China (81027003), and the Research Fund for the Doctoral Program of Ministry of Education of China (20120101130011).

1 Singer W, Gray C M. Visual feature integration and the temporal correlation hypothesis. Annu Rev Neurosci, 1995, 18: 555-586

2 Sanes J N, Donoghue J P. Oscillations in local field potentials of the primate motor cortex during voluntary movement. Proc Natl Acad Sci USA, 1993, 90: 4470-4474

3 Fell J, Klaver P, Lehnertz K, et al. Human memory formation is accompanied by rhinal-hippocampal coupling and decoupling. Nat Neurosci, 2001, 4: 1259-1264

4 Margrie T W, Schaefer A T. Theta oscillation coupled spike latencies yield computational vigour in a mammalian sensory system. J Physiol, 2003, 546: 363-374

5 Kay L M. Theta oscillations and sensorimotor performance. Proc Natl Acad Sci USA, 2005, 102: 3863-3868

6 Lam Y W, Cohen L B, Wachowiak M, et al. Odors elicit three different oscillations in the turtle olfactory bulb. J Neurosci, 2000, 20: 749-762

7 Friedman D, Strowbridge B W. Both electrical and chemical synapses mediate fast network oscillations in the olfactory bulb. J Neurophysiol, 2003, 89: 2601-2610

8 Lagier S, Carleton A, Lledo P M. Interplay between local GABAer- 
gic interneurons and relay neurons generates gamma oscillations in the rat olfactory bulb. J Neurosci, 2004, 24: 4382-4392

9 Beshel J, Kopell N, Kay L M. Olfactory bulb gamma oscillations are enhanced with task demands. J Neurosci, 2007, 27: 8358-8365

10 Gire D H, Schoppa N E. Long-term enhancement of synchronized oscillations by adrenergic receptor activation in the olfactory bulb. $\mathrm{J}$ Neurophysiol, 2008, 99: 2021-2025

11 Chen Q, Xiao L, Liu Q, et al. An olfactory bulb slice-based biosensor for multi-site extracellular recording of neural networks. Biosens Bioelectron, 2011, 26: 3313-3319

12 Aroniadou-Anderjaska V, Ennis M, Shipley M T. Current-source density analysis in the rat olfactory bulb: Laminar distribution of
Kainate/AMPA- and NMDA-receptor-mediated currents. J Neurophysiol, 1999, 81: 15-28

13 Taube J S, Schwartzkroin P A. Mechanisms of long-term potentiation: A current-source density analysis. J Neurosci, 1988, 8: 1645-1655

14 Kulics A T, Cauller L J. Cerebral cortical somatosensory evoked responses, multiple unit activity and current source-densities: Their interrelationships and significance to somatic sensation as revealed by stimulation of the awake monkey's hand. Exp Brain Res, 1986, 62: 46-60

15 Aizenman C D, Kirkwood A, Bear M F. A current source density analysis of evoked responses in slices of adult rat visual cortex: Implications for the regulation of long-term potentiation. Cereb Cortex, 1996, 6: 751-758

Open Access This article is distributed under the terms of the Creative Commons Attribution License which permits any use, distribution, and reproduction in any medium, provided the original author(s) and source are credited. 